\title{
Optical Threshold Layer and Intermediate State Two-Photon PAG Approaches to Double Exposure Lithography
}

\author{
Adam J. Berro ${ }^{\text {a }}$, Xinyu Gu ${ }^{\text {a }}$, Naphtali O’Connor ${ }^{b}$, Steffen Jockusch ${ }^{b}$, Tomoki Nagai ${ }^{\text {a }}$, Toshiyuki \\ Ogata $^{\mathrm{a}}$, Paul Zimmerman ${ }^{\mathrm{c}}$, Bryan J. Rice ${ }^{\mathrm{c}}$, Elizabeth Adolph ${ }^{\mathrm{a}}$, Travis Byargeon ${ }^{\mathrm{a}}$, Jose Gonzalez ${ }^{\mathrm{a}}$, \\ Nicolas J. Turro ${ }^{\mathrm{b}}$, C. Grant Willson ${ }^{*}$ a \\ ${ }^{a}$ Department of Chemistry and Biochemistry, University of Texas at Austin, Austin TX 78712; \\ ${ }^{\mathrm{b}}$ Department of Chemistry, Columbia University, New York, New York, 10027 \\ ${ }^{c}$ SEMATECH, 2706 Montopolis Drive, Austin, Texas, 78741
}

\begin{abstract}
Intermediate state two-photon (ISTP) photoacid generator (PAG) and optical threshold layer (OTL) approaches to double exposure lithography have been explored. We have synthesized "transparent" PAG and sensitizer compounds for use in ISTP systems and have demonstrated the possibility of utilizing such energy transfer systems to generate acid. We have also synthesized side chain liquid crystalline polymers and small molecule azobenzene compounds for use in OTL applications and have begun photoswitching studies.
\end{abstract}

Keywords: double exposure lithography, optical threshold layer, intermediate state two-photon PAG, liquid crystalline polymer, azobenzene

\section{INTRODUCTION}

Since the publication of Moore's law over three decades ago, the semiconductor industry has optimized all three variables in the Rayleigh equation to continue to meet the doubling of transistors on an integrated circuit chip every 18 months. However, delays in extreme ultraviolet lithography (EUV) and the apparent demise of new high index fluids and resists for the extension of immersion lithography have brought to the forefront previously alternative approaches to meet the requirements of 32 and $22 \mathrm{~nm}$ nodes. Two proposals have been receiving attention in this area: double patterning lithography (DPL) and double exposure lithography (DEL). Of these, DEL represents the more cost-effective process, as it eliminates the need to go through the development and etching steps twice, and additionally could allow for both exposures without removing the wafer from the chuck. Simulation studies showed that a two-photon photoacid generator or an optical threshold layer approach was the most promising path to a DEL system. ${ }^{1-3}$ This paper details our results of both of these approaches.

Two-photon PAG materials can be broadly categorized into two groups: simultaneous two-photon absorption materials and sequential two-photon absorption materials. As previously detailed, sequential absorption is more likely to succeed, as it would not require the several orders of magnitude increase in laser pulse power from the exposure tool that would be necessary for simultaneous absorption materials. Our approach to the sequential absorption was to split the PAG functions into separate molecules, one that would absorb the incoming light, convert into a sensitizer, and transfer energy upon the absorption of a second photon, and another that would produce the acid upon energy or electron transfer (Figure 1). By doing this, we hoped to be able to simplify the system to individual components that had the required material properties.

*E-mail: willson@che.utexas.edu; Mail: 1 University Station C0400 Austin, TX, 78712

Advances in Resist Materials and Processing Technology XXVI, edited by Clifford L. Henderson, Proc. of SPIE Vol. 7273, 72731B - (C) 2009 SPIE - CCC code: 0277-786X/09/\$18 - doi: 10.1117/12.814295 


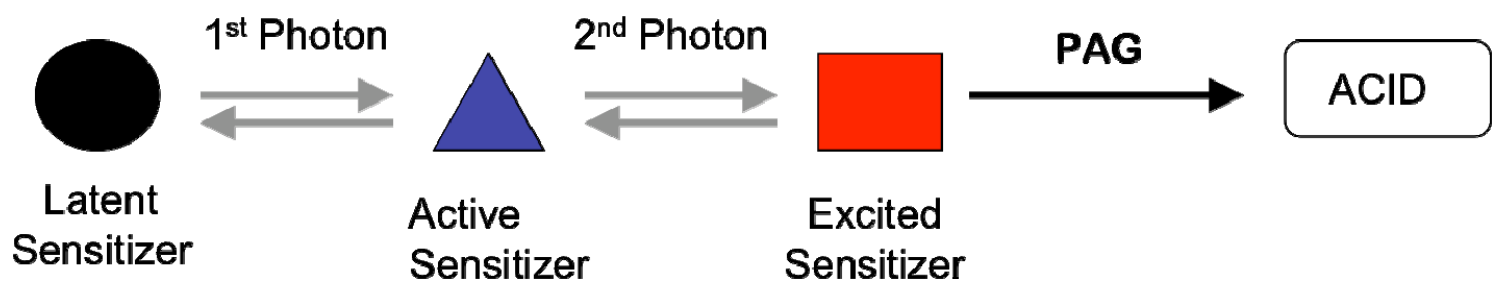

Figure 1: Schematic of ISTP system.

The OTL approach is radically different from a conventional resist system. We envisioned it as involving several layers, one of which would contain a species that could diffuse anisotropically and react in another layer, but is prevented from doing so by a "barrier" layer in the off state. This would require some sort of reversible switch to occur to allow the molecules to pass through. Here, a photoresponsive polymer or system would be required to change its properties based upon the photon interaction from the exposure wavelength and then subsequently "forget" this exposure through either a second exposure of a different wavelength or some other means. Currently, the examples of such threshold behavior exist thermally (i.e. melting and freezing events), but to our knowledge they do not exist photochemically. The heat from the laser pulse itself is not nearly enough to reproducibley melt and freeze the polymer; however if one could incorporate a photochromic material with response at the exposure wavelength, the "melt" of the system could be realized due to part of the polymer altering its configuration, leading to a state change. With this in mind, we sought to synthesize a host liquid crystalline polymer and azobenzene dopant molecules that would exhibit the functions described.

Liquid crystalline polymers have previously been shown to provide distinctly different permeability to gases when in the liquid crystalline state compared to the isotropic liquid state ${ }^{4-6}$ however, such measurements had not been undertaken for solids. Additionally, no work to our knowledge had attempted to produce a lithographic image by diffusing some small molecule from one layer into a different with this process. With this in mind, we began work towards establishing the viability of the aforementioned concepts

\section{EXPERIMENTAL}

All reactions were run in flame-dried glassware under nitrogen atmosphere unless otherwise noted. PAG materials were synthesized as previously reported. ${ }^{7}$ Butyl and hexyl azobenzene dopants (Figure 2) were synthesized according the method of Rondeau et al. ${ }^{8}$ Acetone was dried over molecular sieves and distilled before use. The mesogen for hydrosilylation was synthesized as shown in Figure 3.
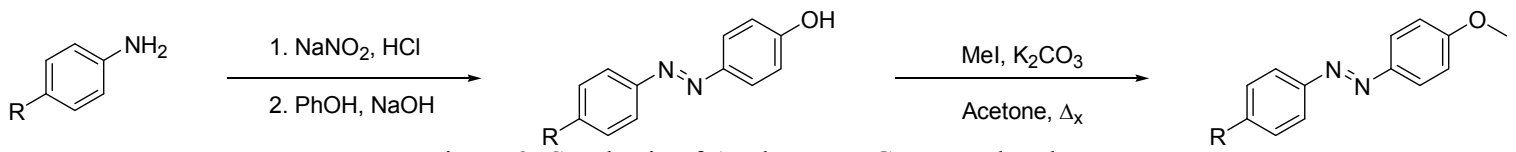

Figure 2: Synthesis of Azobenzene Guest Molecules.

4-allyloxybenzoic acid: A round bottom flask was charged with ethyl 4-hydroxybenzoate (16.61 $\mathrm{g}, 100 \mathrm{mmol})$, allyl bromide $(20 \mathrm{~mL}, 231 \mathrm{mmol}), \mathrm{K}_{2} \mathrm{CO}_{3}(20.73 \mathrm{~g}, 150 \mathrm{mmol})$, potassium iodide $(1.26 \mathrm{~g}, 7.60 \mathrm{mmol})$, and anhydrous DMF $(300 \mathrm{~mL})$. The mixture was stirred under nitrogen at $100^{\circ} \mathrm{C}$ for $24 \mathrm{~h}$, and additional allyl bromide $(20 \mathrm{~mL}, 231 \mathrm{mmol})$ was added and stirred for another $24 \mathrm{~h}$. After being cooled to room temperature, a solution of potassium hydroxide (113.6 g, $2.38 \mathrm{~mol}$, in $300 \mathrm{~mL}$ of $\mathrm{H}_{2} \mathrm{O}$ ) was added and the reaction mixture refluxed for $24 \mathrm{~h}$. After being cooled, the mixture was poured into water and adjusted to $\mathrm{pH} 2-3$. The product, a white precipitate was filtered, washed with water, and dried ( 83\%) ${ }^{1} \mathrm{H}-\mathrm{NMR}\left(400 \mathrm{MHz}, \mathrm{CDCl}_{3}\right): 8.04$ (d, 2H), 6.94 (d, 2H), 6.04 (ddd, 1H), $5.42(\mathrm{~d}, 1 \mathrm{H}), 4.59$ (d, 2H).

4-methoxyphenyl 4-allyloxybenzoate: A round bottom flask was charged with 4-allyloxybenzoic acid (4.306 g, 24.2 mmol) and thionyl chloride $(17.4 \mathrm{~mL}, 242 \mathrm{mmol})$, then refluxed under nitrogen for $5 \mathrm{~h}$. After cooling to room temperature, the remaining thionyl chloride was evaporated in vacuo, then 4-methoxyphenol $(3.00 \mathrm{~g}, 24.2 \mathrm{mmol})$, pyridine $(7.8 \mathrm{~mL}, 96.7 \mathrm{mmol})$, dimethylaminopyridine $(0.183 \mathrm{~g}, 0.967 \mathrm{mmol})$ and $50 \mathrm{~mL}$ of dichloromethane were added. The mixture was stirred at $40{ }^{\circ} \mathrm{C}$ for $24 \mathrm{~h}$. After being cooled, ethyl acetate was added into the reaction mixture, 
and washed with $\mathrm{H}_{2} \mathrm{O}, 1 \mathrm{M} \mathrm{HCl}$, sat. $\mathrm{Na}_{2} \mathrm{CO}_{3}$, and brine. Evaporation gave a white powder, which was recrystallized from ethyl acetate and hexane (80\%) ${ }^{1} \mathrm{H}-\mathrm{NMR}\left(300 \mathrm{MHz}, \mathrm{CDCl}_{3}\right): 8.18(\mathrm{~d}, 2 \mathrm{H}), 7.16(\mathrm{~d}, 2 \mathrm{H}), 7.02(\mathrm{~d}, 2 \mathrm{H}), 6.96(\mathrm{~d}, 2 \mathrm{H})$, $6.10(\mathrm{ddd}, 1 \mathrm{H}), 5.48(\mathrm{~d}, 1 \mathrm{H}), 5.36(\mathrm{~d}, 1 \mathrm{H}), 4.64(\mathrm{~d}, 2 \mathrm{H}), 3.82(\mathrm{~s}, 3 \mathrm{H})$.

poly((3-(4-(4-methoxyphenyloxycarbonyl)phenyloxy)propyl)methyl siloxane): A round bottom flask was charged with 4-methoxyphenyl 4-allyloxybenzoate $(3.00 \mathrm{~g}, 10.5 \mathrm{mmol})$, polyhydromethylsiloxane (0.632 g), platinum (0) divinyltetramethyldisiloxane $(0.3 \mathrm{~mL}, 0.1 \mathrm{M}$ solution in xylene, $0.02 \mathrm{mmol})$ and $20 \mathrm{~mL}$ of anhydrous toluene. The mixture was stirred under nitrogen at $50{ }^{\circ} \mathrm{C}$ for $24 \mathrm{~h}$, then 1-decene was added and stirred 6 more hours to quench remaining $\mathrm{Si}-\mathrm{H}$. The mixture was filtered using celite and reprecipitated with THF and methanol (57\%).

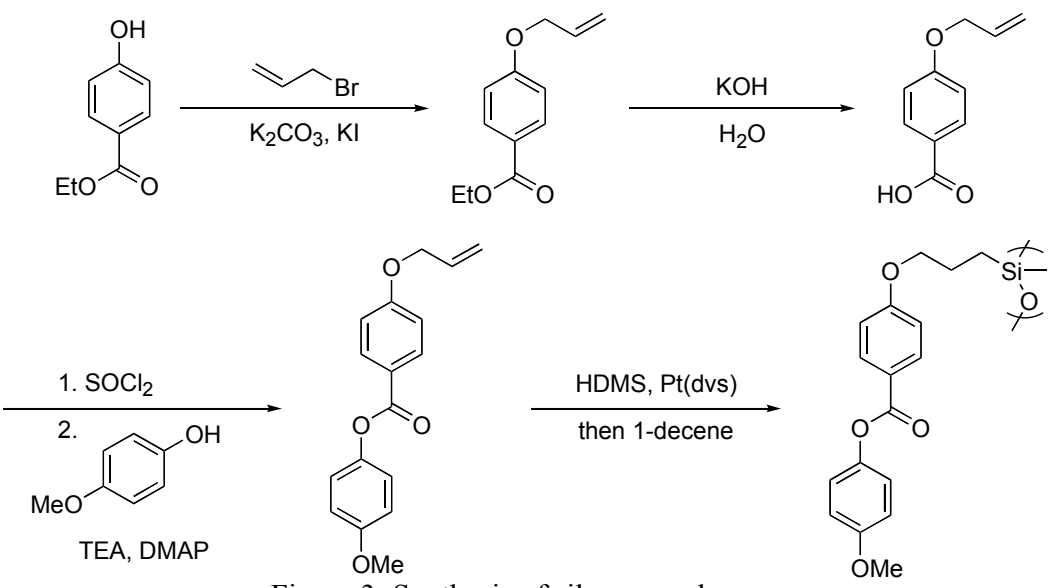

Figure 3: Synthesis of siloxane polymer.

Acrylate monomers were synthesized according to a literature procedure as shown in Figure 4. ${ }^{9}$ Polymerization was conducted as follows: the acrylate monomer was added to a flask along with benzoyl peroxide ( 2 mol\%) and dissolved in THF $(0.25 \mathrm{M}$ with respect to monomer). Three freeze pump thaw cycles were performed and the reaction was placed into a preheated $60{ }^{\circ} \mathrm{C}$ oil bath and stirred $15 \mathrm{~h}$. The mixtures were then cooled and precipitated into ethyl ether and filtered.<smiles>O=C([O-])c1ccc(O)cc1</smiles>
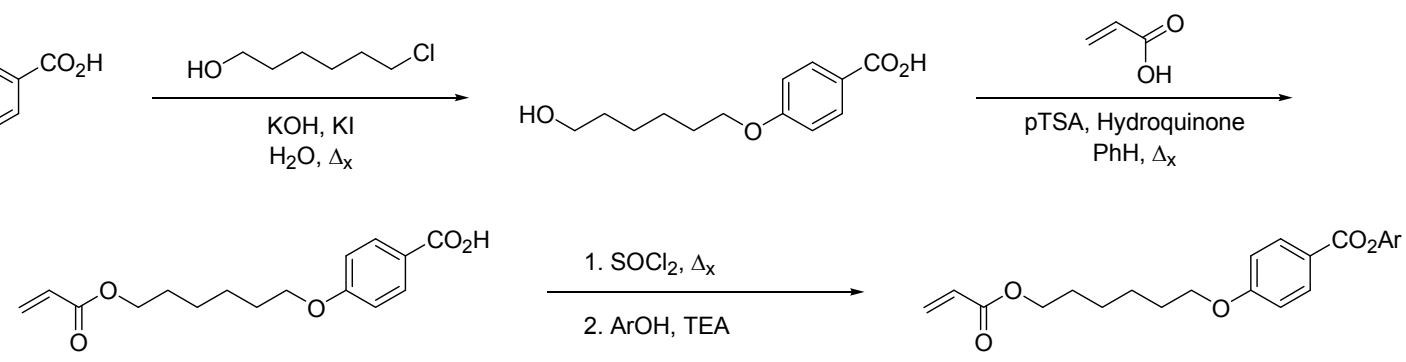

Figure 4: Synthesis of acrylate monomers.

$\mathrm{ArOH}=\mathrm{PhOH}, p-\mathrm{OMePh}$

\section{INSTRUMENTATION}

Gel Permeation Chromatography was performed using an Agilent autosampler and pump connected to a Viscotek column heater and refractometer using Viscotek columns with THF as the mobile phase. Differential scanning calorimetry was performed with a TA Instruments Q100 DSC. Polarized optical microscopy used a Mettler FP80 controller, FP82 HT hotstage and an Olympus BX60 microscope. Exposures were performed with a Novacure high intensity mercury arc lamp through a light guide fitted with $365 \mathrm{~nm}$ or $436 \mathrm{~nm}$ bandpass filters. For exposures at 248 nm, a Cymer excimer laser equipped with a light guide was used. Ellipsometry data was collected with a J. A. Woolam M-2000D ellipsometer using CompletEASE software. Temperature of the films and wafer were controlled using a 
Peltier device connected to a Newport Model 3040 Temperature controller module. Film thickness measurements were made using a Veeco Dektak 6M profilometer.

\section{RESULTS}

\subsection{Intermediate Stage Two-Photon PAG}

For the ISTP system, the first goal was to synthesize a PAG that would not absorb at the operational wavelength. This led us to norbornane type PAG compounds as shown in (Figure 5).<smiles>CCON1C(=O)C2C3CCC(O3)C2C1=O</smiles>

Figure 5: "Transparent" PAG structures.

As previously reported, ${ }^{7}$ these compounds exhibit low absorbance down to $220 \mathrm{~nm}$ (Figure 6). Indeed, even formulations using either of the synthesized PAG compounds in a standard $193 \mathrm{~nm}$ resin exposed at $193 \mathrm{~nm}$ with an excimer laser did not produce an image until the dose energy began to degrade the polymer without the PAG present.

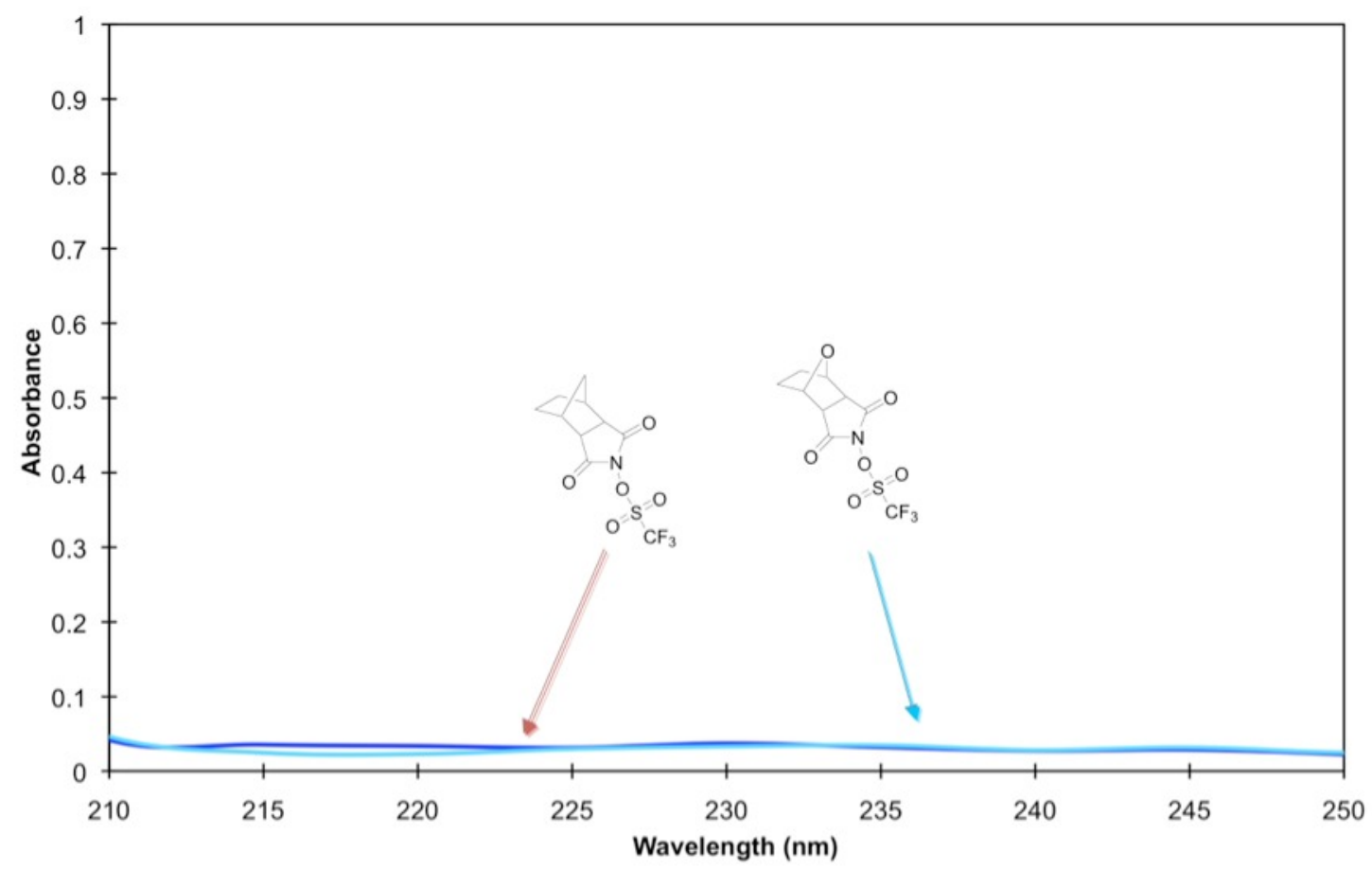

Figure 6: Absorption of "transparent" PAG materials in Acetonitrile (1mM).

With this result, we set out to find a suitable sensitizer molecule that would allow for acid generation. Molecular modeling using Spartan indicated that substituted naphthalenes, particularly 2-methoxynaphthalene, would fall into a range capable of conducting electron transfer to the PAG material, whereas similar anthracene-based sensitizers would be unable to do so. Through a series of experiments, our modeling data proved to be accurate, as no tested anthracenebased compound was able to produce acid from our synthesized PAG materials. Gratifyingly, 2-methoxynaphthalene

proved to be a good candidate and generated acid upon exposure to $254 \mathrm{~nm}$ light as indicated by fluorescein dye fluorescence (Figure 7). The amount of acid generated was quantified by integration of the fluorescence peak associated with the fluoroscein. 

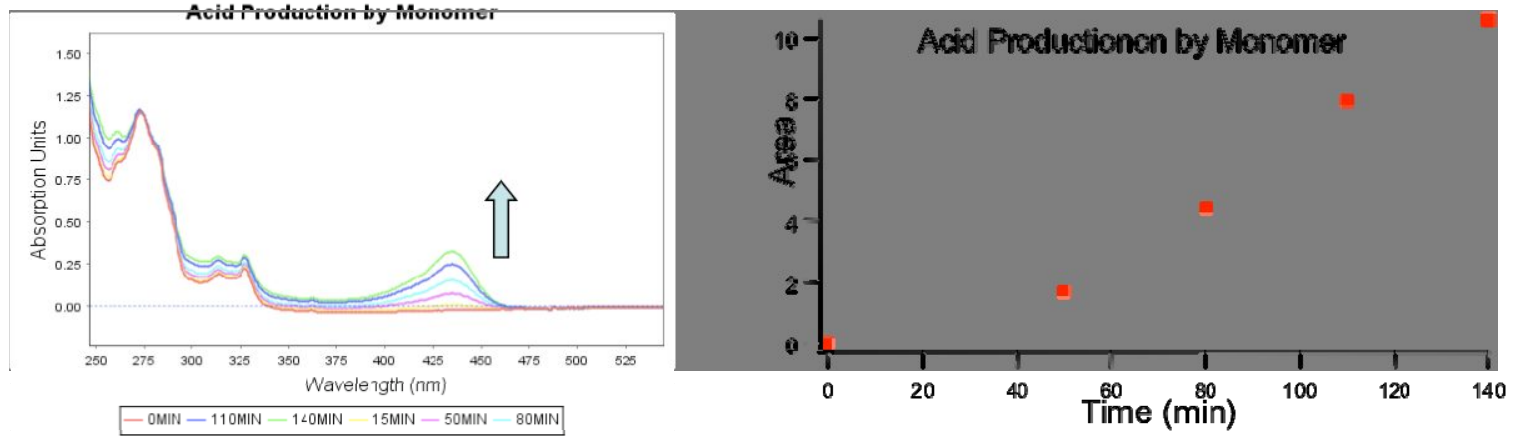

Figure 7: Acid generation from monomeric 2-methoxynaphthalene.

To provide additional information about the nature of the reaction, exposures were also conducted in the presence of benzophenone, a triplet sensitizer as well as with sodium iodide, a known electron scavenger. In both cases, no acid was observed, providing evidence that electron transfer occurs through an excited singlet state from the naphthalene.

With a suitable sensitizer molecule in hand, we then attempted to make a system that could demonstrate the required nonlinear dose response. Conveniently, 2-methoxynaphthalene is known to dimerize with UV exposure, allowing an opportunity to achieve our goal. Using the same experimental setup as was used for the single-photon acid generation,

we were able to generate acid (Figure 8). Efforts are ongoing to further develop this system, to quantify the dose response, and to determine the degree of nonlinearity. To improve the efficiency of the system, efforts are being focused on synthesizing various tethered dimers related to the system with the hope of linking not only the two naphthalene components, but the acid generation component as well, which would not only increase the acid yield, but simplify resist formulation. We believe that tethering the components will be key to the success of such a system, as it eliminates the diffusion of the two naphthalene components from one another. With the PAG tethered to the sensitizer, there is no need to take diffusion effects into account in the system. Synthetic work in this area continues in our labs.
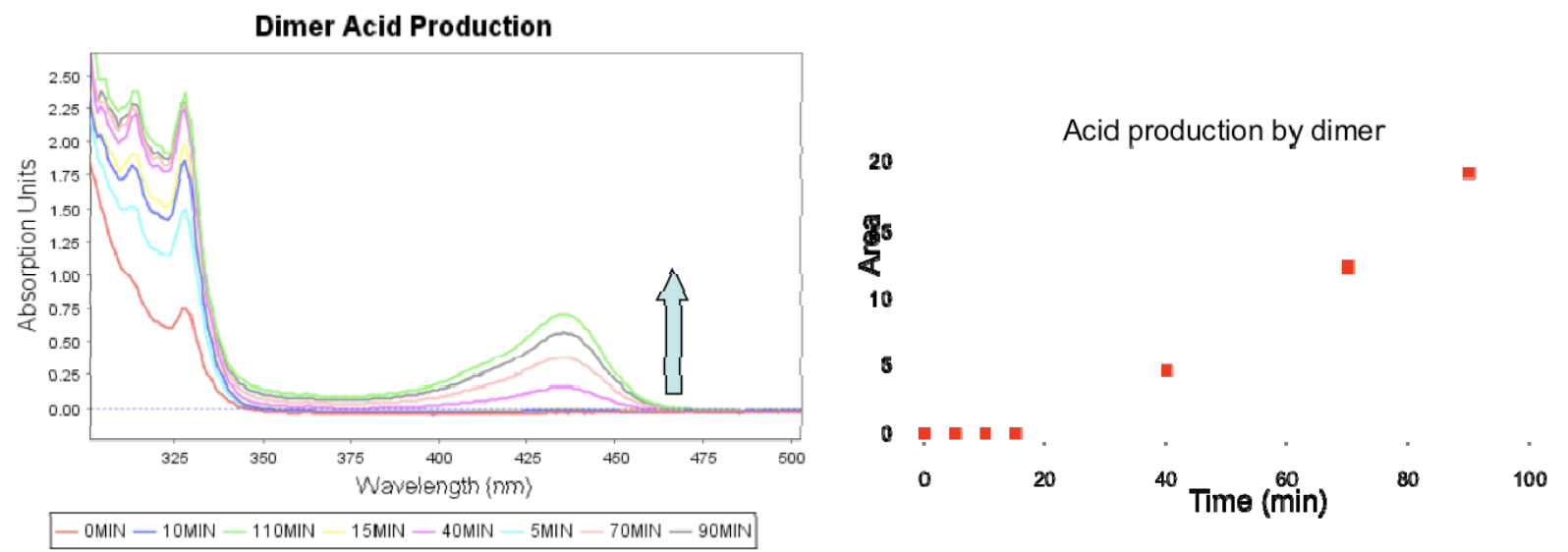

Figure 8: Acid production from dimeric 2-methoxynaphthalene.

\subsection{Optical Threshold Layer Approach}

For the OTL approach, we initially synthesized an azobenzene-containing liquid crystalline polymer (Figure 9) ${ }^{10}$ to answer the question of whether or not diffusion could be successfully modulated by altering the state of the barrier layer. 


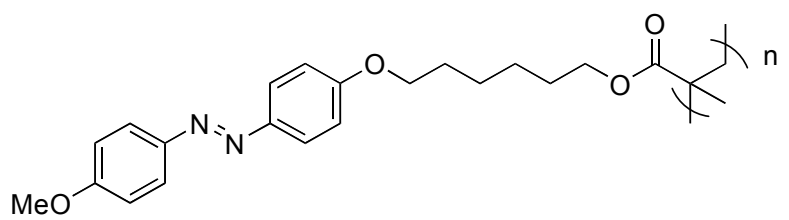

Figure 9: Azobenzene Polymer.

Our initial tests looked at the change in permeability accompanied by the state change from liquid crystal to isotropic liquid, and if observed, the move to a photoswitching state change. For this, we used a film stack as shown in Figure 10.

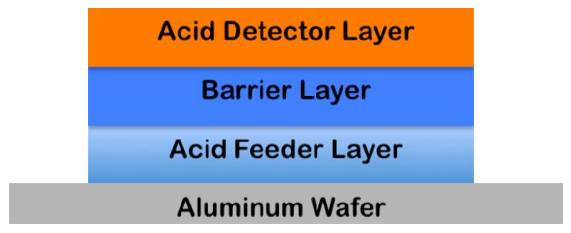

Figure 10: Film stack for diffusion measurements.

The detector layer of poly( $t$-Boc-styrene), when exposed to acid loses $\mathrm{CO}_{2}$, a change that is readily observable in the infrared absorbance spectrum. In this system, our feeder layer contained a PAG (TPS-OTf). After generating acid through exposure at $248 \mathrm{~nm}$, we could test the time required to diffuse the acid through the barrier layer by monitoring for the disappearance of the carbonyl peak in the IR spectrum. For a liquid crystal to isotropic transition at $125^{\circ} \mathrm{C}$, a rather dramatic increase in the diffusion rate can be seen from just below to just above this temperature (Figure 11).

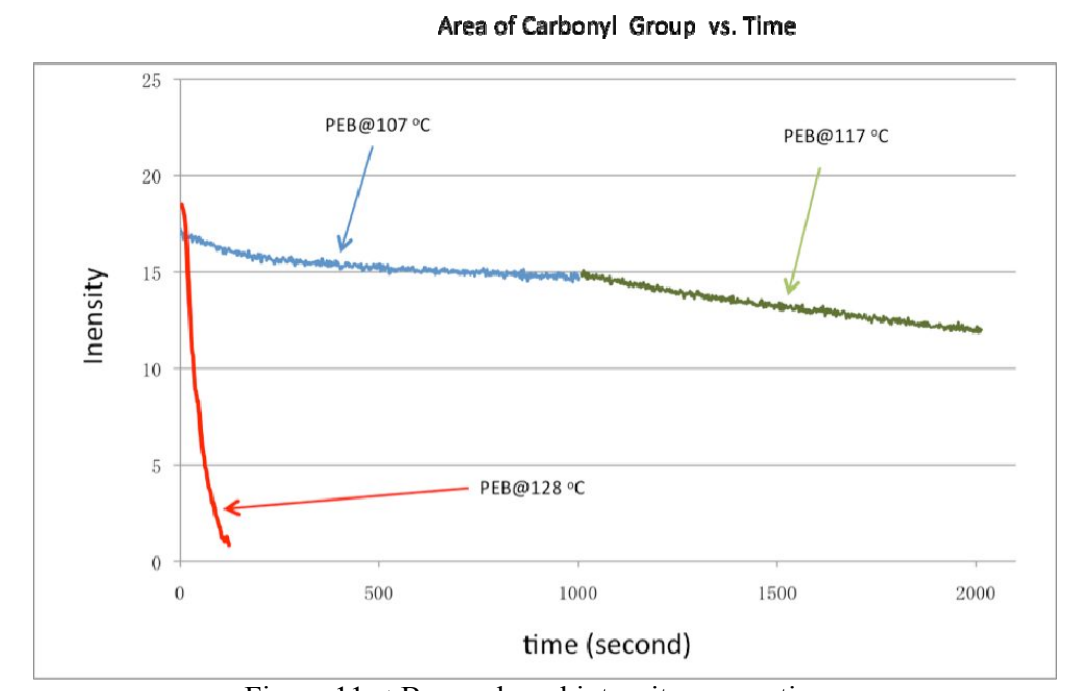

Figure 11: $t$-Boc carbonyl intensity versus time.

Due to the high transition temperatures of this polymer, photoswitching of the azobenzene and its effect on the physical state of the material could not be tested. The isomerization back to its original trans form is too rapid at temperatures above $70^{\circ} \mathrm{C}$. Hense, we began to pursue other polymers, which ultimately led to a polymer that we would dope with the azobenzene photochemically active compound. This allowed a wider range of polymers to be used, as well as previously synthesized compounds that already had transition temperatures near room temperature. The synthesis of the desired alkene for hydrosilylation to the purchased siloxane polymer afforded reasonable quantities of the desired product. The hydrosilylation reaction was conducted, and the remaining Si-H bonds were reacted with 1-decene to provide the completely reacted polymer. With the desired materials in hand, attention was turned to polymer properties. Polarized optical microscopy (POM) was used in conjunction with differential scanning calorimetry (DSC) to determine the liquid crystalline range of the siloxane polymer. 


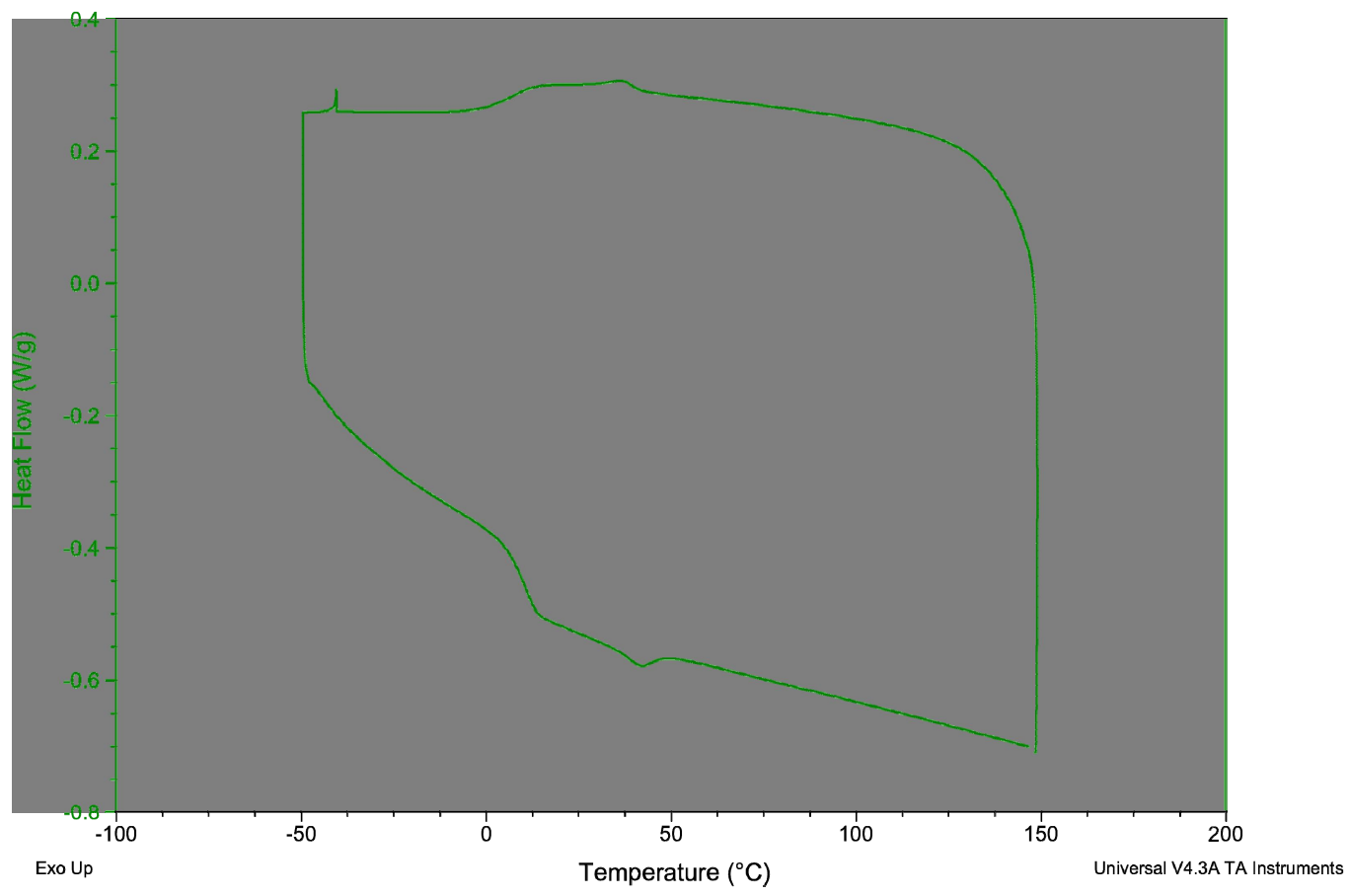

Figure 12: DSC data for siloxane polymer.

The initial system was composed of a 1:9 by weight mixture of the azobenzene guest to siloxane polymer. A thin film of this mixture was determined to be liquid crystalline from below room temperature up to $38{ }^{\circ} \mathrm{C}$, which represents a lowering of the clearing temperature by about $8{ }^{\circ} \mathrm{C}$. With the observed change in the clearing temperature of the resultant mixture, the film-coated wafer coupons were exposed to $365 \mathrm{~nm}$ illumination $\left(105 \mathrm{~mJ} / \mathrm{cm}^{2}\right.$ exposure dose) through a contact mask. The coupon was then placed on a polarizing optical microscope, so that only the liquid crystalline areas could be observed. As shown in Figure 13, the liquid crystalline layer was disrupted in the exposed portion, leaving a dark area lacking texture. This indicates that the azobenzene guest molecules have isomerized sufficiently to act as an impurity and disrupted the liquid crystalline order.

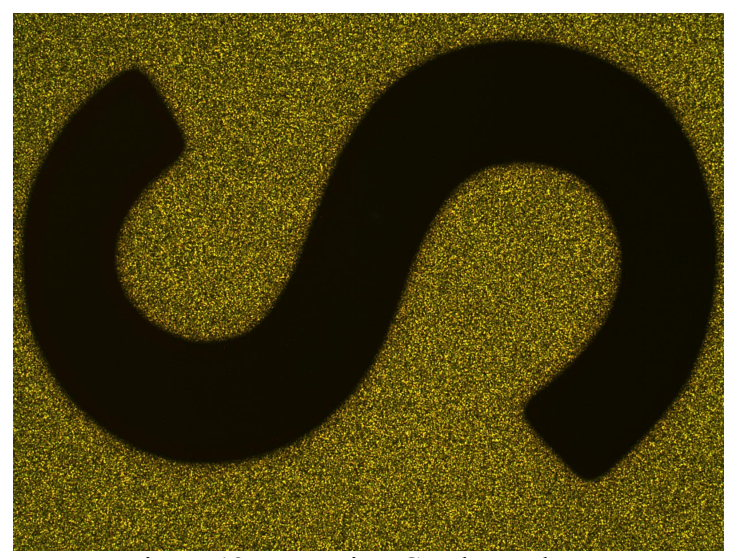

Figure 13: Image in LC polymer layer.

Having successfully produced an image via exposure, the next step was to efficiently reverse the exposure. Using 436 $\mathrm{nm}$ light through a bandpass filter and a second contact mask, some reversal of the isomerization is observed, with a much greater difference seen after annealing at $40{ }^{\circ} \mathrm{C}$ (Figure 14). 


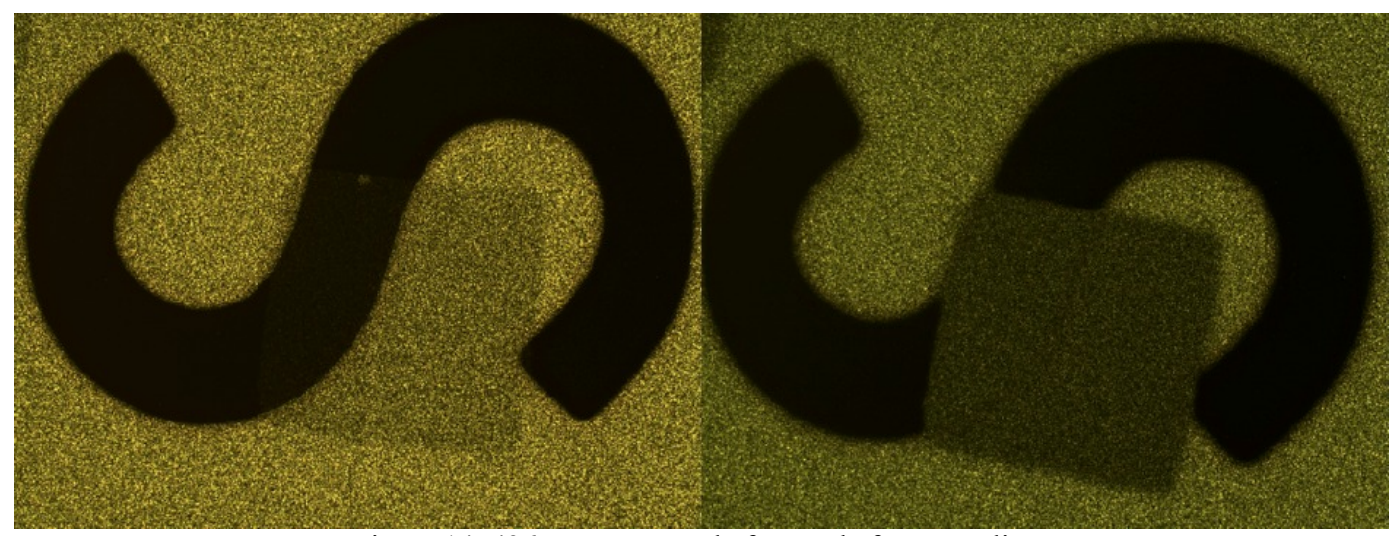

Figure 14: $436 \mathrm{~nm}$ exposure before and after annealing.

Acrylate based liquid crystalline polymers were also pursued in hope that these doped systems would allow for easier measurement and film stack generation, as the resulting mixture had less liquid character. The lack of a para alkoxy substituent on the mesogen afforded a polymer that was liquid crystalline at room temperature (Figure 15).

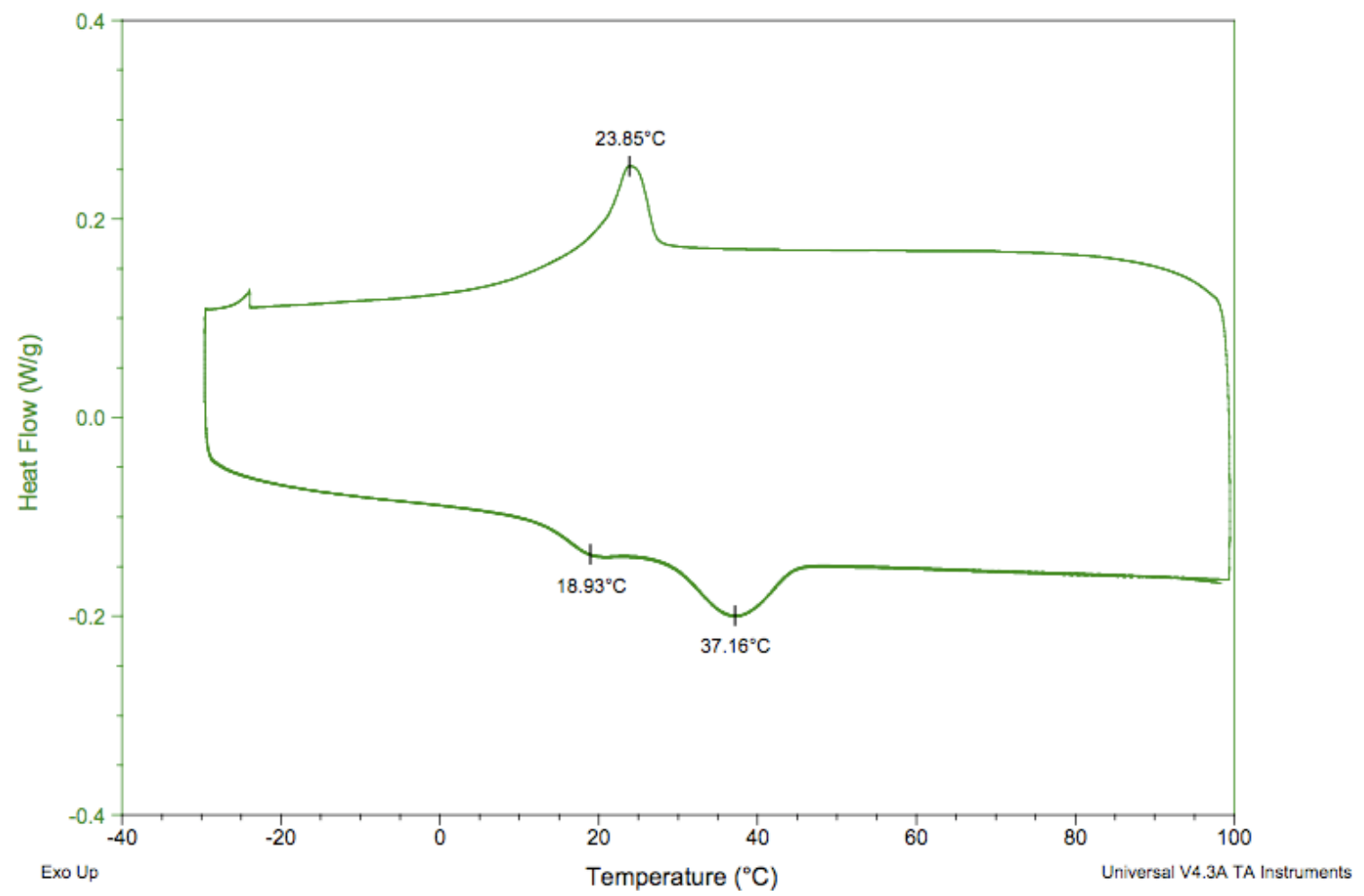

Figure 15: DSC data from acrylate polymer.

As before, a mixture of 1:9 by weight mixture of azobenzene guest molecule:polymer was made. The solution was cast onto a glass slide using dichloromethane. The film was subjected to POM analysis which showed that the material transitioned from liquid crystalline to isotropic liquid between 39 and $40{ }^{\circ} \mathrm{C}$ (Figure 16). 

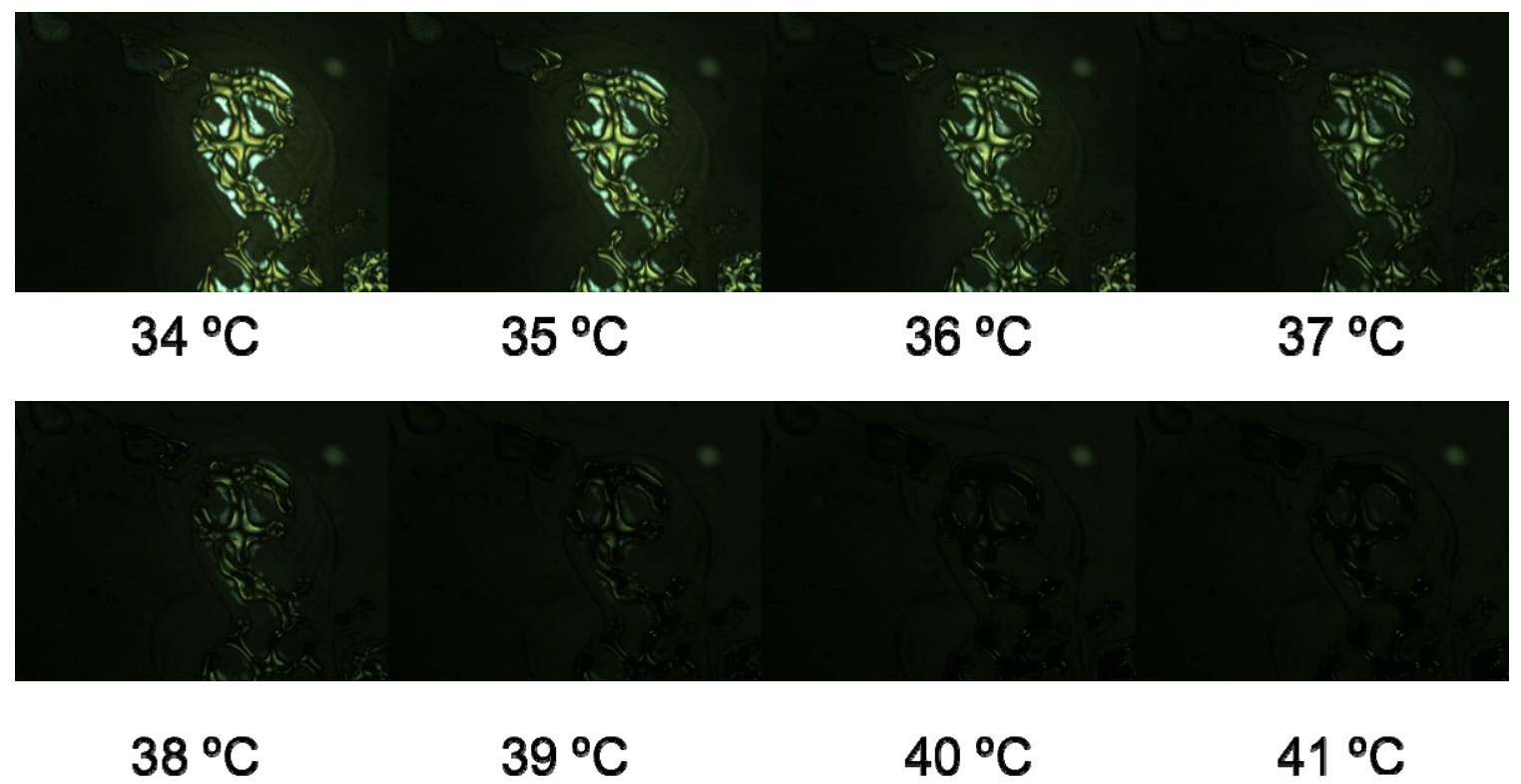

$39^{\circ} \mathrm{C}$

$40^{\circ} \mathrm{C}$

$41^{\circ} \mathrm{C}$

Figure 16: POM images of doped acrylate polymer.

\section{CONCLUSION}

For two-photon photoacid generators, we have established that the functions of the PAG can be decoupled into an absorbing component and an acid-generating component, which opens up additional material prospects. Synthesis continues to provide even lower absorbance acid-generating components, and the search for additional sensitizer compounds is ongoing. We believe that tethering the two species that will form the latent sensitizer as well as linking the PAG to this species will prove to be crucial to the success of these materials.

While the above experiments were conducted at a $365 \mathrm{~nm}$ exposure wavelength, the basic principles that apply to such a radically redesigned photoresist can apply to a system at lower wavelengths using different photoresponsive groups in the same polymer matrix. The above results show that a liquid crystalline polymer whose permeability can be altered by switching to the isotropic liquid state can serve as a barrier to diffusion. We have also shown that with exposure to 365 $\mathrm{nm}$ light, an azobenzene-doped liquid crystalline compound can be photochemically changed to the isotropic liquid state and then with subsequent $436 \mathrm{~nm}$ exposure and annealing can reform the LC phase. Our group continues to work on the photochemical permeability switch for these materials. The success of such a system will then spawn work into new photochromics active at $193 \mathrm{~nm}$ wavelengths that could then be doped into the current polymer systems. However, very little information is available on compounds that have photochromic properties at $193 \mathrm{~nm}$; therefore, more research must be conducted for our approach to an OTL material to be viable.

\section{ACKNOWLEDGEMENTS}

The authors would like to thank Elizabeth Costner from the Willson Group for help in measuring absorbance values of two-photon materials and Robert Bristol of Intel for helpful discussions and collaboration in addition to the SEMATECH Engineering Research Center for funding. SEMATECH and the SEMATECH logo are registered service marks of SEMATECH, Inc. All other service marks and trademarks are the property of their respective owners. 


\section{REFERENCES}

[1] Lee, S.; Byers, J.; Jen, K.; Zimmerman, P.; Rice, B.; Turro, N. J.; Willson, C. G. “An analysis of double exposure lithography options." Proc. SPIE, 6924, 69242A-1-12, (2008).

[2] Byers, J.; Lee, S.; Jen, K.; Zimmerman, P.; Turro, N. J.; Willson, C. G. "Double exposure materials: simulation study of feasibility." J. Photopolym. Sci. Technol. 20(5), 707-717 (2007).

[3] Lee, S.; Jen, K.; Byers, J.; Zimmerman, P.; Turro, N. J.; Willson, C. G. "Materials Modeling and Development for Use in Double Exposure Applications.” J. Micro/Nanolithogr. MEMS MOEMS 8(1), In Press (2009).

[4] Weinkauf, D. H.; Paul, D. R. "Gas Transport Properties of Thermotropic Liquid Crystalline Copolyesters. I. The Effects of Orientation and Annealing." J. Polym. Sci., Part B: Polym. Phys. 30, 817-835 (1992).

[5] Weikauf, D. H.; Paul, D. R. "Gas Transport Properties of Thermotropic Liquid Crystalline Copolyesters. II. The Effects of Copolymer Composition.” J. Polym. Sci., Part B: Polym. Phys. 30, 837-849 (1992).

[6] Chiou, J.S.; Paul, D. R. "Gas Transport in a Thermotropic Liquid-crystalline Polyester.” J Polym. Sci., Part B: Polym. Phys. 25, 1699-1707 (1987).

[7] O'Conner, N. A.; Berro, A. J.; Lancaster, J. R.; Jockusch, S.; Nagai, T.; Ogata, T.; Lee, S.; Zimmerman, P.; Willson, C. G.; Turro, N. J. "Toward the Design of a Sequential Two Photon Photoacid Generator for Double Exposure Photolithography." Chem. Mater. 20 (24), 7374-7376 (2008).

[8] Rondeau, R. E.; Berwick, M. A.; Steppel, R. N.; Serve, M. P. “Central linkage influence upon mesomorphic and electrooptical behavior of diaryl nematics. General proton magnetic resonance method employing a lanthanide shift reagent for analysis of isomeric azoxybenzenes.” J. Am. Chem. Soc. 94 (4), 1096-1102 (1972).

[9] Horvath, J.; Nvitrai, K.; Cser, F.; Hardy, G. "Polymerization in Liquid Crystal - XVII: Effect of Flexible Substituents in the Mesogenic Side-Chain on the Behaviour of Mesogenic Polymers." Eur. Polym. J. 21 (3), 251257 (1985).

[10] Sadagopan, K.; Rekha, A. S.; Ratna, D.; Samui, A. B. "Synthesis and characterization of azo-based methacrylate polymers with methoxy and nitro end groups for nonlinear optics applications." J. Appl. Polym. Sci. 104 (6), 34973504 (2007). 